\title{
Fully carbon metasurface: Absorbing coating in microwaves
}

D. Bychanok, S. Li, G. Gorokhov, K. Piasotski, D. Meisak, P. Kuzhir, E. A. Burgess, C. P. Gallagher, F. Y. Ogrin, A. P. Hibbins, A. Pasc, A. Sanchez-Sanchez, V. Fierro, and A. Celzard

Citation: Journal of Applied Physics 121, 165103 (2017); doi: 10.1063/1.4982232

View online: http://dx.doi.org/10.1063/1.4982232

View Table of Contents: http://aip.scitation.org/toc/jap/121/16

Published by the American Institute of Physics 


\title{
Fully carbon metasurface: Absorbing coating in microwaves
}

\author{
D. Bychanok, ${ }^{1, a)}$ S. Li, ${ }^{2}$ G. Gorokhov, ${ }^{1}$ K. Piasotski, ${ }^{1}$ D. Meisak, ${ }^{1}$ P. Kuzhir, ${ }^{1}$ E. A. Burgess, ${ }^{3}$ \\ C. P. Gallagher, ${ }^{3}$ F. Y. Ogrin, ${ }^{3}$ A. P. Hibbins, ${ }^{3}$ A. Pasc, ${ }^{2}$ A. Sanchez-Sanchez, ${ }^{4}$ V. Fierro, ${ }^{4}$ \\ and A. Celzard ${ }^{4}$ \\ ${ }^{1}$ Research Institute for Nuclear Problems Belarusian State University, 11 Bobruiskaya str., 220030 Minsk, \\ Belarus \\ ${ }^{2}$ NANO Group, SRSMC - UMR Université de Lorraine - CNRS 7565, Vandoeuvre-lès-Nancy Cedex, France \\ ${ }^{3}$ University of Exeter, Exeter EX4 4QL, United Kingdom \\ ${ }^{4}$ Institut Jean Lamour - UMR Université de Lorraine - CNRS 7198, ENSTIB, 27 rue Philippe Seguin, \\ CS 60036, 88026 Epinal Cedex, France
}

(Received 3 February 2017; accepted 12 April 2017; published online 26 April 2017)

\begin{abstract}
The microwave-absorbing properties of a heterostructure consisting of an ordered monolayer of porous glassy carbon spheres were experimentally and theoretically investigated in the Ka-band (26-37 GHz) frequency range. The electromagnetic response of such a "moth-eye"-like all-carbon metasurface at a normal incidence angle was modelled on the basis of long-wave approximation. Modelling parameters in the Ka-band were used to estimate and predict the absorption properties of monolayers in free space in the range $1-40 \mathrm{GHz}$. Experimental and theoretical results demonstrate that a metasurface based on porous glassy carbon spheres is an inert, lightweight, compact, and perfectly absorbing material for designing new effective microwave absorbers in various practically used frequency ranges. Published by AIP Publishing. [http://dx.doi.org/10.1063/1.4982232]
\end{abstract}

\section{INTRODUCTION}

Until now, artificial metamaterials proposed by Pendry et $a l^{1}{ }^{1}$ and realised experimentally by Smith $e t a .^{2}$ were always hybrids based on dielectric and metal elements of sophisticated geometries. Recently, Liu et al. ${ }^{3}$ have proposed a fully dielectric metasurface which has some advantages, including high absorption of electromagnetic (EM) radiation of particular frequency by a very small volume and simple geometry. In the present paper, the alternative idea and the practical realisation of an all-carbon metasurface made of glassy carbon spheres (GCSs) are presented. Such GCSs were simply organised in periodic arrays and could provide a perfect absorption of microwaves. The individual GCSs have an average diameter about $\sim 1 \mathrm{~mm}$ and consist of porous glassy carbon. In terms of pore size, their internal structure is in-between that of carbon foams ${ }^{4-6}$ and aerogels. ${ }^{7}$ Glassy carbon is a non-graphitizing, highly disordered carbon with features that make it definitely different from graphite: it is hard and brittle with a conchoidal fracture, shiny, and isotropic, and with a density typically $30 \%$ lower than that of graphite. Its electrical conductivity is isotropic and may be as high as $30 \times 10^{3} \mathrm{~S} / \mathrm{m}$ after pyrolysis at $3000^{\circ} \mathrm{C}^{8}$ This material is commonly used for producing inert and thermally stable electrodes in electrochemistry. ${ }^{9}$ The combination of good electrical conductivity of glassy carbon and the spherical shape can be very useful for practical applications, particularly for those related to the absorption of electromagnetic (EM) radiation. Many researchers worldwide are concentrated on the design of broadband absorbers. Generally, the following mechanisms of EM absorption are used for practical applications: ferromagnetic resonance, ${ }^{10,11}$ Ohmic losses, ${ }^{12,13}$ dipole

\footnotetext{
a)dzmitrybychanok@ya.ru. URL:www.nano.bsu.by
}

relaxation, ${ }^{14}$ and lossy scattering in metamaterials. ${ }^{15}$ At present, many groups focus on the investigation of carbon-based polymer composites for developing effective microwave absorbers. ${ }^{16-18}$ In this paper, we considered the features of normal scattering of microwave radiation on an ordered monolayer of porous carbon spheres on a Perfect Electrically Conducting (PEC) plate, schematically shown in Fig. 1.

Materials based on spherical particles are attracting interest of many research groups worldwide. In the recent decade, there has been a considerable effort devoted to the investigation of electromagnetic properties of similar structures, including metasurfaces formed by planar arrays of spherical particles ${ }^{19}$ and lossless magneto-dielectric spheres embedded in a background matrix, ${ }^{20,21}$ as well as twodimensional lossy heterostructures. ${ }^{22}$ In most of the cases, the investigations involved modelling based on the earlier work of Lewin ${ }^{23}$ for sparse arrays of spherical particles. In turn, the materials considered here are formed by highly lossy glassy carbon spheres ordered in a close-packed array. In our recent report, ${ }^{12}$ we considered a similar structure but

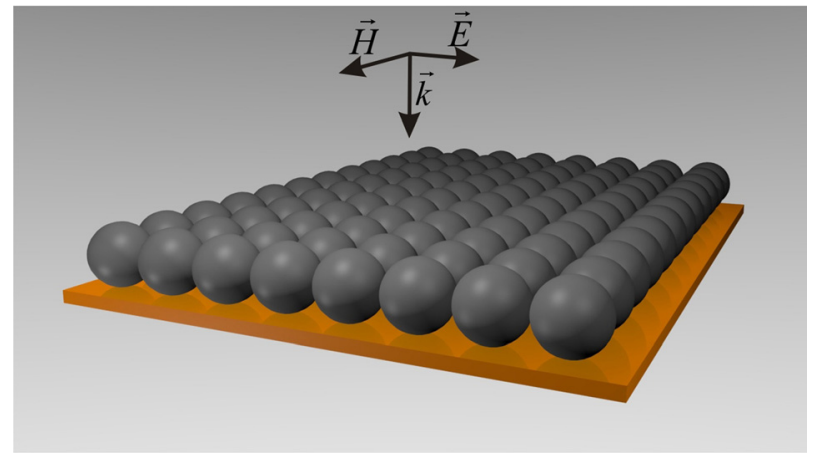

FIG. 1. Monolayer of porous glassy carbon spheres on a PEC plate. 
based on hollow carbon spheres (HCSs). Compared to the latter, the GCSs are much more mechanically resistant, which is especially important for further practical use.

The analysis of the electromagnetic response of nonuniform periodic structures may be significantly simplified when the wavelength of the external electromagnetic wave is much larger than the characteristic lateral dimensions of the structure. Moreover, non-homogeneous structures with a graded refractive index are widely used in the industry for designing and developing various types of microwave absorbers $^{24-29}$ and the long-wave approximation ${ }^{30-33}$ is commonly applied to describe and predict their electromagnetic properties in the microwave frequency range.

The present paper is organized as follows. The details of preparation of GCSs and of the characterisation techniques as well of experimental microwave measurements are given in Section II. In Section III, we provide the material's main characteristics. In Section IV, we describe the homogenisation technique which is used to obtain the equivalent material properties and the electromagnetic response of the GCS monolayer. Herein, we also compare the experimental data with the results of modelling. Theoretical predictions of the absorption properties in free space of the GCS-based metasurface are discussed in Section V. Finally, the Conclusions section summarises the findings and gives an outlook on potential application of such metasurfaces.

\section{EXPERIMENTAL}

\section{A. Preparation of materials}

The synthesis of porous glassy carbon spheres is based on several main components including sodium alginate (Algin Texturas), calcium chloride (Roth), resorcinol (Alfa Aesar), and a 37\% aqueous solution of formaldehyde (Roth). All these chemicals were used "as is" without further purification. First, a $20 \mathrm{ml}$ aqueous solution containing $12.8 \mathrm{~g}$ resorcinol, $9.43 \mathrm{~g}$ formaldehyde solution, and $2.22 \mathrm{~g}$ of $\mathrm{CaCl}_{2}$ was prepared. Dropwise addition of $5 \mathrm{ml}$ of a $0.5 \mathrm{wt} \%$ sodium alginate in water, using a syringe of volume $10 \mathrm{ml}$ having a needle of diameter and length $0.8 \mathrm{~mm}$ and $50 \mathrm{~mm}$, respectively, to the former solution heated at $60^{\circ} \mathrm{C}$ under magnetic stirring, instantaneously induced gelation and led to polymer beads of size about $2.1 \mathrm{~mm}$. The beads were recovered by filtration after $1 \mathrm{~h}$ of stirring at the same temperature of $60^{\circ} \mathrm{C}$, and then treated in hot pressurised water $\left(180^{\circ} \mathrm{C}, 24 \mathrm{~h}\right)$ for hydrothermal carbonisation (HTC) in an autoclave. The aim of HTC was to stabilise the spheres and increase their carbon content. Finally, the beads recovered after $\mathrm{HTC}$ were submitted to pyrolysis at $900{ }^{\circ} \mathrm{C}\left(2{ }^{\circ} \mathrm{C} / \mathrm{min}\right.$, $2 \mathrm{~h}$ dwell time).

Whereas the size of the final carbon spheres did not significantly depend on the experimental conditions, their density could be tuned through the concentration of the reactants. Given the carbon precursor used here, i.e., resorcinolformaldehyde resin, the spheres were made of glassy carbon, a material rather similar to the one with which hollow spheres were prepared from another non-graphitizing precursor and reported recently for EM applications. ${ }^{12}$

\section{B. Characterisation of materials}

Carbon spheres were observed without preliminary metallisation with a FEI Quanta 600 FEG scanning electron microscope (SEM). Elemental analysis was performed with an elemental analyser Vario EL Cube (Elementar). The contents of $\mathrm{C}, \mathrm{H}, \mathrm{N}$, and $\mathrm{S}$ were measured through the combustion of 3 identical samples in a quartz tube at $1150{ }^{\circ} \mathrm{C}$ under helium and oxygen in excess, and the average was calculated for each element. The oxygen content was estimated by difference.

Raman spectroscopy was carried out using an XploRa Raman spectrometer (Horiba) without sample preparation. The spectra were collected under a microscope using a $50 \times$ objective. The Raman-scattered light was dispersed by a holographic grating with 1200 lines/mm and detected by a CCD camera. Three lasers were used, having wavelengths of 532, 638, and $785 \mathrm{~nm}$, thus corresponding to incident energies of $2.33,1.94$, and $1.58 \mathrm{eV}$, respectively. The lasers were filtered at $10 \%$ of their nominal power for avoiding any heating or damage of the samples. Each spectrum was obtained by accumulation of 2 spectra recorded from 800 to $2200 \mathrm{~cm}^{-1}$ over $120 \mathrm{~s}$. It was systematically checked that no significant difference was observed when the analysis was performed in different areas of the same sample, suggesting the excellent homogeneity of the materials.

Finally, the textural properties were determined from nitrogen adsorption-desorption isotherms at $-196^{\circ} \mathrm{C}$, using an ASAP 2020 surface area analyser (Micromeritics). The samples were out-gassed overnight under vacuum at $250^{\circ} \mathrm{C}$ prior to analysis. Micropore volume, $V_{\mu}$, and surface area, $S_{B E T}$, were determined by application of Dubinin-Radushkevich and Brunauer-Emmett-Teller (BET) methods, respectively. The total pore volume measurable by adsorption, $V_{T}$, was defined as the volume of liquid nitrogen corresponding to the amount adsorbed at a relative pressure $P / P_{0}=0.99$. The mesopore volume, $V_{m}$, was calculated as the difference $V_{T}-V_{\mu}$. The pore size distribution was determined by application of the nonlinear density functional theory (NLDFT).

\section{Ka-band measurements}

The electromagnetic response of a GCS-based monolayer was experimentally investigated in the Ka-band $(26-37 \mathrm{GHz})$ using a scalar network analyser ELMIKA R2-408R. All measurements were carried out in a $7.2 \times 3.4 \mathrm{~mm}$ waveguide system. For testing the model presented below in Section III, two types of experiments were performed. In one type, the GCSs were initially packed to form a close-compact single layer which was then carefully fixed inside the waveguide between two $1 \mathrm{~mm}$-thick layers of Styrofoam ${ }^{\circledR}$ (Dow Chemical), a trademarked brand of closed-cell extruded polystyrene foam transparent to microwave. Due to the unavoidable, slight distribution of GCS sizes and due to some defects in the packing, the as-obtained 2D ordering was closer to a square-shaped lattice. The electromagnetic response of the GCS-based monolayer was obtained as amplitude ratios of transmitted to input $\left(S_{21}\right)$ and reflected to input $\left(S_{11}\right)$ signals. In the second type of experiment, the layer of Styrofoam behind the sample was replaced by the PEC-plate (i.e., behaving as a mirror with a 
reflection coefficient of $100 \%$ ). In that case, only the $S_{11^{-}}$ parameters were measured. Reflection and transmission coefficients were then obtained from S-parameters: $R=S_{11}^{2}$ and $T=S_{21}^{2}$, respectively. The absorption coefficient was then calculated as $A=1-R-T$ and $A=1-R$ in the first and second types of experiments, respectively.

\section{MATERIAL'S CHARACTERISTICS}

Scanning Electron Microscopy (SEM) images of typical samples of GCSs are presented in Figs. 2(a) and 2(b). A photo of the GCS monolayer formed "as is" is presented in Fig. 2(c). The order of real GCS-arrays is in between cubic or hexagonal.

From SEM analysis, the average diameter of the spheres was found to be around $d=0.79 \mathrm{~mm}$. The inner structure was relatively homogeneous and close to that of glassy carbon aerogels with a typical pore size less than $1 \mu \mathrm{m}$, as evidenced by SEM images at higher magnification (not shown). Based on the average diameter and on the weight of a number of carbon spheres, their average density was found to be $\rho=0.39 \mathrm{~g} / \mathrm{cm}^{3}$. Taking a value of $1.55 \mathrm{~g} / \mathrm{cm}^{3}$ for the skeletal density of glassy carbon, ${ }^{12,34}$ the porosity of the GCS was estimated to be $75 \%$.

The elemental weight composition was the following: $91.70 \pm 0.50 \%$ carbon; $0.80 \pm 0.15 \%$ hydrogen; $0.21 \pm 0.10 \%$ nitrogen; and $0.00 \%$ sulphur. The deduced oxygen content was then around $7 \%$. Those values are quite typical for a carbon material derived from phenolic resin and other oxygenated precursors such as those reported in the synthesis. ${ }^{34,35}$ As a result, the carbon was expected to be highly disordered, hence the name "glassy" it was given, and this was proved by Raman spectra shown in Fig. 3.

It can indeed be seen in Fig. 3 that the spectrum obtained at an energy of $2.33 \mathrm{eV}$ presents a broad $\mathrm{D}$ band centred on $1340 \pm 2 \mathrm{~cm}^{-1}$ and a slightly narrower $\mathrm{G}$ band of rather similar intensity at $1595 \pm 2 \mathrm{~cm}^{-1}$. A shallow valley between them and a shoulder at around $1200 \mathrm{~cm}^{-1}$, accounted by contributions called D3 and D4, respectively, are also observed. Besides, the positions of D and $\mathrm{G}$ bands, their general shapes, and their FWHM values (close to 135 and $70 \mathrm{~cm}^{-1}$, respectively) fully match those already reported for glassy carbon derived from phenolic/furanic resins pyrolysed below $1000{ }^{\circ} \mathrm{C}$ (see for instance ${ }^{36-40}$ ). Decreasing the laser energy, $E_{L}$, from 2.33 to $1.58 \mathrm{eV}$ produced the expected phenomena, i.e., a shift of the D band to lower wavenumbers, whereas the $\mathrm{G}$ band remained at the same position but with a relatively lower intensity. Although only three incident energies were available for establishing quantitative trends, the intensity ratio of $\mathrm{D}$ to $\mathrm{G}$ bands was found to vary as $I_{D} / I_{G} \simeq E_{L}^{-1.13}$, and the $\mathrm{D}$ band was seen to shift with the increasing excitation energy at a rate of $48 \mathrm{~cm}^{-1} / \mathrm{eV}$. The latter values and the aforementioned ones (band positions and widths) are consistent with each other and are typical of highly disordered carbons having crystallite size close to-or lower than $-5 \mathrm{~nm},{ }^{41}$ an order of magnitude already reported for similar carbon materials. $^{35}$

Although the texture of such carbon is highly disordered, the electrical conductivity remains rather high, around
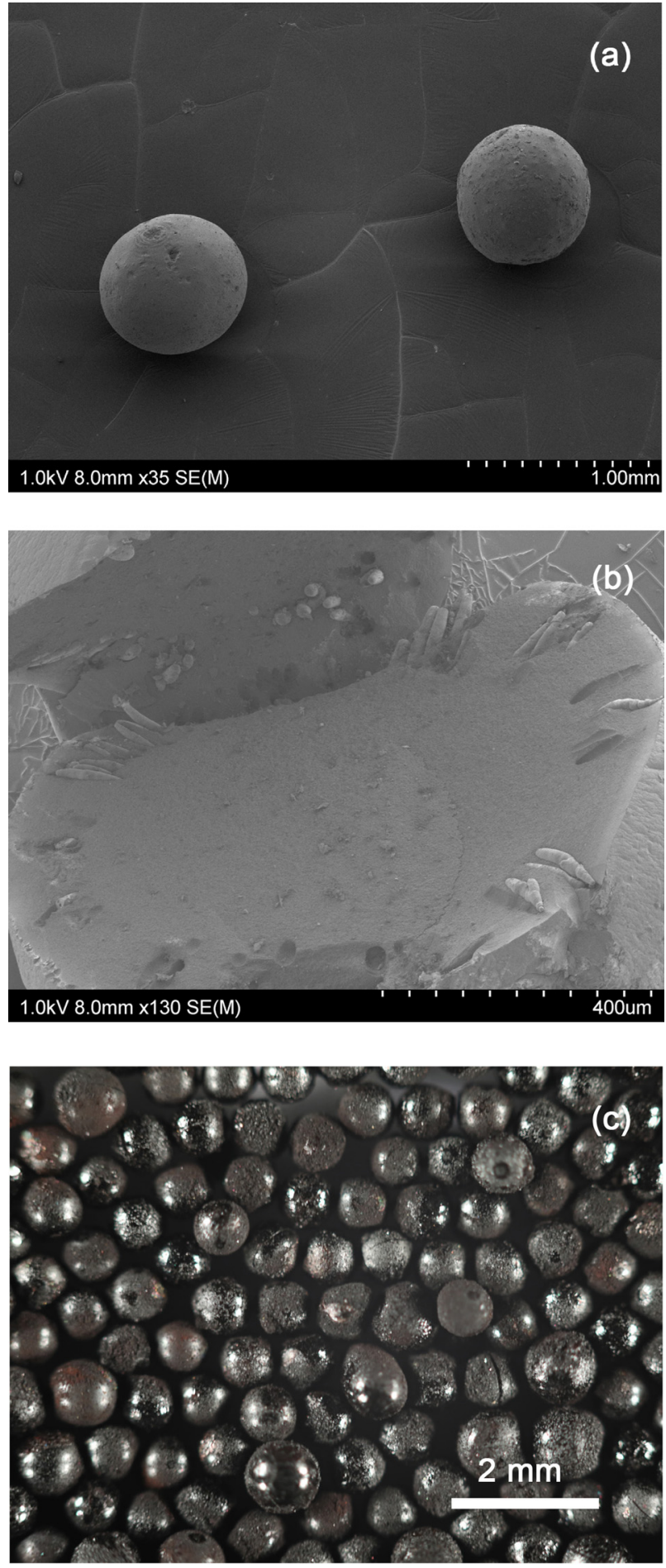

FIG. 2. SEM images of: (a) porous glassy carbon spheres, and (b) inner structure of a broken sphere; (c) Photo of a real GCS monolayer.

$200 \mathrm{~S} / \mathrm{m}$, see Section IV. This value is lower but still consistent with the one reported elsewhere for non-porous glasslike carbon pyrolysed at $900{ }^{\circ} \mathrm{C}$, around $7000 \mathrm{~S} / \mathrm{m} .{ }^{36}$ The present material indeed has a porosity $\Phi=75 \%$ and, assuming that the simplest relationship for the conductivity of metal foams holds, ${ }^{42}$ i.e., $\sigma=\sigma_{\text {bulk }}(1-\Phi)^{3 / 2}$, then the conductivity is one order of magnitude lower than that of the bulk. The present conductivity value is even lower than that, for at least two additional reasons expected to decrease the conductivity further: (i) the presence of alginate of natural origin in the 


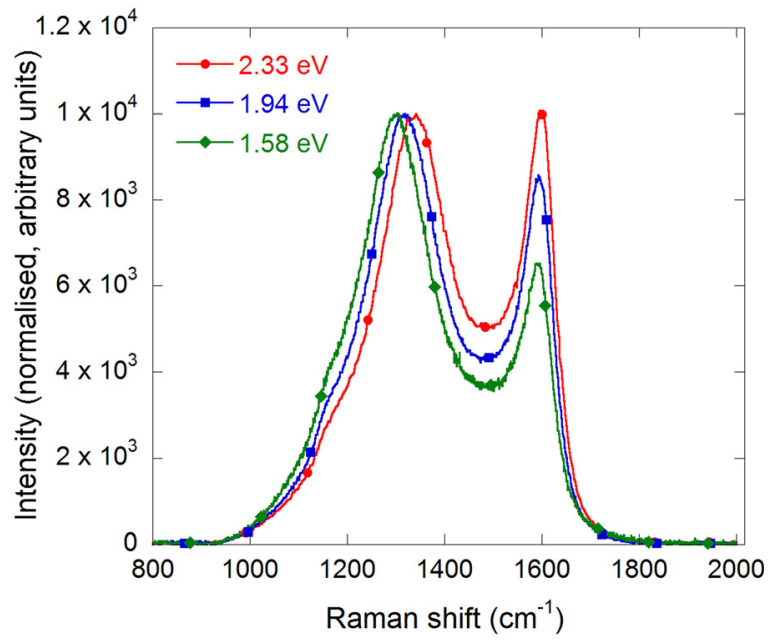

FIG. 3. First-order Raman spectra of glassy carbon spheres as a function of laser incident energy.

synthesis should have introduced impurities in the carbon matrix and (ii) the porosity is far narrower than that of a foam, in favour of a much higher scattering of charge carriers within the material.

Indeed, the adsorption-desorption given in Fig. 4 is typical of a highly microporous solid (pore size $<2 \mathrm{~nm}$ ) with a very low amount of mesoporosity $(2-50 \mathrm{~nm})$, as confirmed by the pore-size distribution shown in the inset. Moreover, the corresponding surface area was $S_{B E T}=588 \mathrm{~m}^{2} / \mathrm{g}$, whereas micropore and mesopore volumes were $V_{\mu}=0.22 \mathrm{~cm}^{3} / \mathrm{g}$ and $V_{m}=0.04 \mathrm{~cm}^{3} / \mathrm{g}$, respectively. As a result, the macropore volume $(>50 \mathrm{~nm})$ was therefore deduced to be predominant and equal to $1 / \rho-1 / \rho_{c}-V_{m}-V_{\mu}=0.75 \mathrm{~cm}^{3} / \mathrm{g}$.

\section{MODELLING}

The wavelength of the radiation in the $\mathrm{Ka}$-band $(1 \mathrm{~cm}$ at $30 \mathrm{GHz}$ ) exceeds considerably the GCS diameter. Hence, the

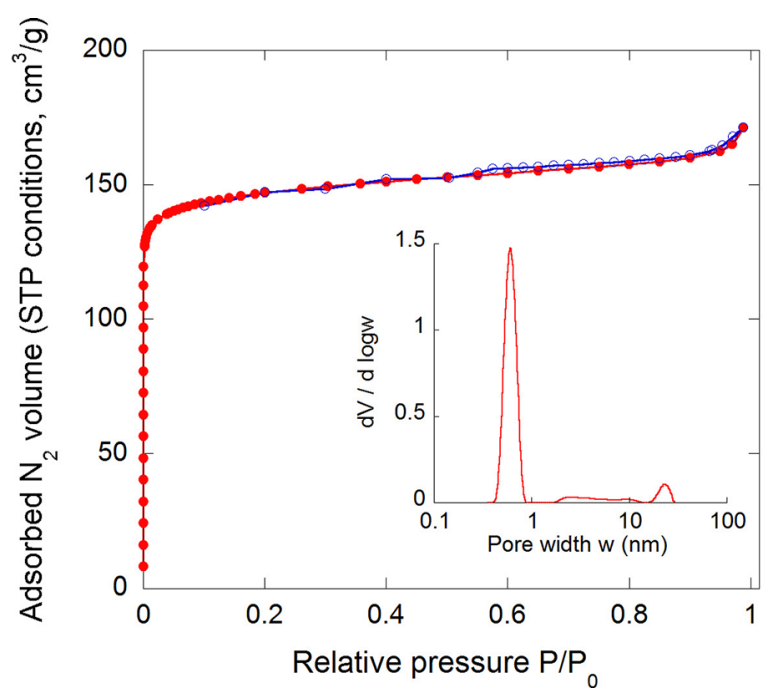

FIG. 4. Adsorption-desorption isotherm (full and empty symbols, respectively) of glassy carbon spheres, and the corresponding pore-size distribution (inset). calculations can be significantly simplified by applying a homogenisation procedure to the given structure and using the long-wave approximation. This methodology is traditionally used in optics for modelling antireflective coatings, ${ }^{30,32}$ and was successfully applied in our recent works. ${ }^{12,27}$

It is important to note that the primitive homogenisation used in Ref. 12 for a monolayer of hollow spheres, consisting of averaging non-conductive air regions with highly conductive carbon regions according to their relative volume fractions, was not successful in describing the experimental data in the case of the present porous spheres. Simple averaging indeed gave correct results in the case of hollow sphere monolayer because the shell thickness of HCSs was much smaller than their diameter, and because the effective dielectric permittivity in the middle part of the layer remained constant. For a monolayer made of GCSs, the spatial distribution of effective dielectric permittivity is much more non-uniform; thus, the homogenisation procedure had to be modified to account for these variations.

To model the electromagnetic response of an ordered monolayer of conducting spherical particles on a metal surface, we followed homogenisation methods which are widely used for designing microwave absorbers. ${ }^{24,26,28}$ These methods are generally based on the application of equivalent circuits for calculating the effective dielectric permittivity of the structure, and are valid for a wide range of absorber geometries. After homogenisation, the electromagnetic response of the investigated structure in the long-wave approximation was considered to be equivalent to that of a layer of bulk material of the same thickness but with graded effective dielectric permittivity.

\section{A. Homogenisation procedure}

The general principles of the homogenisation procedure used in this work are detailed in Refs. 24 and 28. Throughout the article, we use SI units and assumed an $\exp [i \omega t-i k z]$ harmonic time convention.

Let us consider the side projection of a GCS monolayer along the xz-plane as shown in Fig. 5(a). The origin of the coordinates is located in the middle of the monolayer. The wave vector of the initial radiation is parallel to the $\mathrm{x}$ direction. Fig. 5(b) presents the monolayer cross-section along the yz-plane at the coordinate $x(|x|<D / 2)$, where $D$ is the GCS diameter. This cross-section consists of roundlike carbon regions of radius $r=\sqrt{D^{2} / 4-x^{2}}$ and air regions. Due to the symmetry of the system, only one quarter of the unit cell (the square of side $D / 2$ and thickness $d x$ presented in Fig. 5(c)) may be considered to calculate the effective dielectric permittivity of the layer at coordinate $x$. Let us examine a thin slice of it, having a thickness $d y$. It consists of carbon and air regions, which are equivalent to two capacitors in series (Fig. 5(d)). The stack of such carbon-air capacitors in parallel contributes to the effective capacitance of the layer. Now, this parameter may be easy calculated by integration over the $y$-coordinate within the unit cell and then converted into effective dielectric permittivity of the layer at coordinate $x$ 
(a)

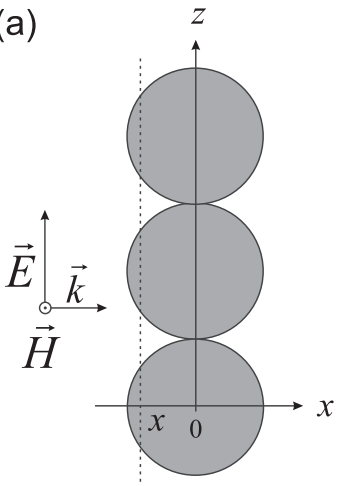

(c) $\sqrt{\frac{1}{4} D^{2}-x^{2}}$

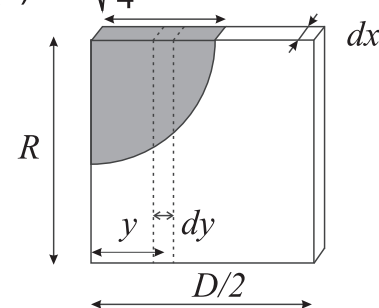

$z$ (b)

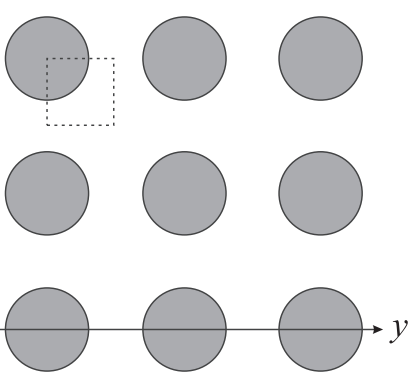

(d)

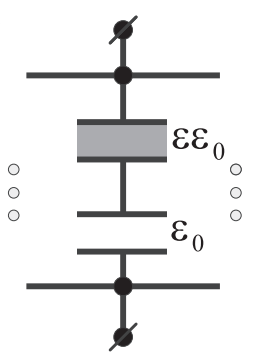

FIG. 5. (a) Side projection of a GCS monolayer along the xz-plane; (b) cross-section along the yz-plane at the coordinate $x(|x|<D / 2)$; (c) quarter of unit cell used for the calculation of the equivalent material constants; (d) equivalent capacitance model of the layer.

$$
\begin{aligned}
\varepsilon_{e f f}(x, \nu)= & \frac{D-\sqrt{D^{2}-4 x^{2}}}{D}+\int_{0}^{\sqrt{\frac{D^{2}}{4}-x^{2}}} \\
& \times \frac{\varepsilon(\nu) d y}{\varepsilon(\nu)\left(\frac{D}{2}-\sqrt{\frac{D^{2}}{4}-x^{2}-y^{2}}\right)+\sqrt{\frac{D^{2}}{4}-x^{2}-y^{2}}},
\end{aligned}
$$

where $\varepsilon(\nu)=1-\frac{i \sigma}{2 \pi \nu \varepsilon_{0}}$ is the dielectric permittivity of glassy carbon, $i$ being the imaginary unit, $\sigma=200 \mathrm{~S} / \mathrm{m}$ is the quasistatic bulk electrical conductivity, $\nu$ is the frequency, and $\varepsilon_{0}=8.85 \times 10^{-12} \mathrm{~F} / \mathrm{m}$ is the permittivity of vacuum. The value of $\sigma$ was obtained by fitting the experimental data below, and seems realistic for porous glassy carbon with density $\rho=0.39 \mathrm{~g} / \mathrm{cm}^{3}$ since it is in good agreement with recent published results. ${ }^{6,43}$ The spatial distribution of effective dielectric permittivity (1) calculated at $30 \mathrm{GHz}$ is presented in Fig. 6.

From Fig. 6, we can see that the absolute values of both real and imaginary parts of effective $\varepsilon$ are rather low in the outer regions of the GCS monolayer, and increase towards the centre. Nevertheless, in the middle region where the glassy carbon fraction is high and where the contribution of conductive losses dominates, the real part of effective $\varepsilon$ decreases and has a minimum in the centre of the monolayer. It should be noted that this complex distribution of dielectric permittivity is formed by combination of insulating $(\varepsilon=1)$ and conducting $\left(\varepsilon(\nu)=1-\frac{i \sigma}{2 \pi \nu \varepsilon_{0}}\right)$ media. It is also interesting to see that, if we apply Eq. (1) to a monolayer of lossless dielectric spheres, for example with $\varepsilon=2$, we obtain a spatial distribution very close to the parabolic one used in Refs. 12 and 30 .

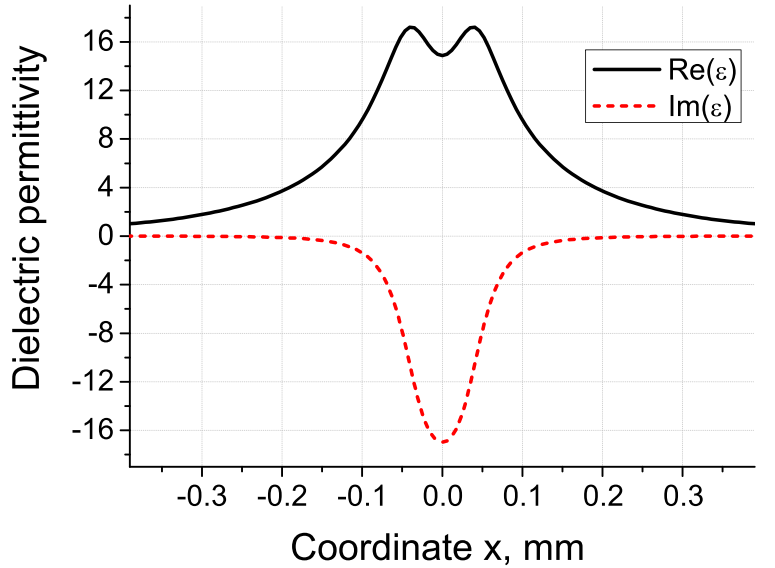

FIG. 6. Spatial distribution of the homogenised effective dielectric permittivity of the GCS-based monolayer calculated using Eq. (1) at $30 \mathrm{GHz}$.

To conclude this section, we should point out that the homogenisation procedure presented above is valid as far as the wavelength of the initial radiation considerably exceeds the GCS diameter. In this case, we can assume that the periodic GCS-based monolayer structure (or GCS metasurface) presents the same electromagnetic response as an effective medium with a spatial distribution of effective dielectric permittivity (1).

\section{B. Multi-layered medium}

For calculating the EM response of a structure having the spatial dispersion described by Eq. (1), we used the model of wave distribution in a multi-layered medium..$^{31,44}$

Let us consider a layer of bulk material with the spatial dispersion obeying Eq. (1). For calculating its coefficients of reflection $R$, transmission $T$, and absorption $A$, the following procedure was applied. ${ }^{12}$ The layer was first divided into $N$ parts. When $N$ is high, it can be assumed that the refractive index inside each part is constant.

Using Maxwell equations, it is possible to obtain the direct solutions for electric and magnetic fields at the boundaries of each thin layer. Written in matrix notations, they have the following form: ${ }^{12,31,44}$

$$
\left[\begin{array}{l}
E_{1} \\
H_{1}
\end{array}\right]=\left[\begin{array}{ll}
\cos \left(k_{t} \tau_{0}\right) & i \sin \left(k_{t} \tau_{0}\right) k_{0} / k_{t} \\
i \sin \left(k_{t} \tau_{0}\right) k_{t} / k_{0} & \cos \left(k_{t} \tau_{0}\right)
\end{array}\right]\left[\begin{array}{l}
E_{2} \\
H_{2}
\end{array}\right],
$$

where $E_{1}, H_{1}$ and $E_{2}, H_{2}$ are electric and magnetic fields in the left and right sides of a layer of thickness $\tau_{0}$, respectively, $k_{t}=\frac{2 \pi}{\lambda a} \sqrt{n(x)^{2} a^{2}-\lambda^{2} / 4}$ and $k_{0}=\frac{2 \pi}{\lambda a} \sqrt{n_{0}^{2} a^{2}-\lambda^{2} / 4}$ are the wave vectors in the layer of refractive index $n(x)$ and in air (refractive index $n_{0}=1$ ) inside the waveguide of width $a=7.2 \mathrm{~mm}$, respectively, $\lambda$ being the wavelength. The first term in the right part of Eq. (2) is known as the characteristic matrix of the layer.

In the case of a multi-layered structure, the characteristic matrix of the whole layer is the product of all single layer matrices. The $R$ and $T$ coefficients of the N-layered subsystem inside the waveguide can be calculated as 


$$
\begin{aligned}
& R=S_{11}^{2}=\left(\frac{n_{0}-C / B}{n_{0}+C / B}\right)^{2}, \\
& T=S_{21}^{2}=\left(\frac{2 n_{0}}{n_{0} B+C}\right)^{2},
\end{aligned}
$$

where

$$
\left[\begin{array}{l}
B \\
C
\end{array}\right]=\left\{\prod_{t=1}^{N}\left[\begin{array}{ll}
\cos \left(k_{t} \tau\right) & i \sin \left(k_{t} \tau\right) k_{0} / k_{t} \\
i \sin \left(k_{t} \tau\right) k_{t} / k_{0} & \cos \left(k_{t} \tau\right)
\end{array}\right]\right\}\left[\begin{array}{l}
1 \\
n_{0}
\end{array}\right] .
$$

For the numerical calculations, we used $n(x, \nu)=\sqrt{\varepsilon_{e f f}(x, \nu)}$ to obtain the refractive index inside the GCS-monolayer region $|x|<D / 2$.

The aforementioned equations were applied in Section IV $\mathrm{C}$ to the description and to the analysis of the experimental data.

\section{Comparison between experiment and modelling}

The electromagnetic response of the GCS-based monolayer without a metal plate, either measured in waveguide or modelled in the Ka-band, is given in Fig. 7(a). From this figure, it can be seen that the free-standing monolayer absorbed about $24 \%$ of the initial radiation power. However, the absorption properties of the monolayer could be significantly improved when a metal plate is located beneath the monolayer. The corresponding $S_{11}$-parameters are presented in Fig. 7(b). In this case, the absorption coefficient $A=1-S_{11}^{2}$ indeed varied in the range 30\%-94\%. Additionally, the interference minimum of the signal reflected from the GCSmonolayer could be shifted by varying the width of the air gap between the monolayer and the metal plate (Fig. 7(c)). The combination of the GCS-based monolayer, an air gap $(l=6.1 \mathrm{~mm})$, and a metal plate thus allows observing experimentally an asymmetric and sharp absorption peak with a maximum at about $A=84 \%$. The position of this peak was quite well predicted by the modelling.

In Figs.7(a)-7(c), a good agreement can be observed between experiments and modelling for the three independent measurements. The maximum absolute difference between experimental data and results of theoretical modeling in Figs. 7(a), 7(b), and 7(c) is 0.09, 0.04, and 0.27, respectively. We can see that the best correlation between experiment and modeling is for the GCS monolayer located on a metal plate. When the GCS is in the form of a monolayer separated from the metal plate by an air gap of width $6.1 \mathrm{~mm}$, the divergence near $33 \mathrm{GHz}$ is maximal but the shape of the peak and its position still are in good agreement with the modelling. The possible reason for some deviation between modelled and measured S-parameters in Fig. 7 is the inhomogeneity of diameters of the real porous GCSs forming the monolayer. Nevertheless, the obvious coincidence between experiment and theory confirms the relevance of the model presented here, and allows using it to predict the electromagnetic response as well as the absorption properties in free space and in a broader frequency range, as discussed below.

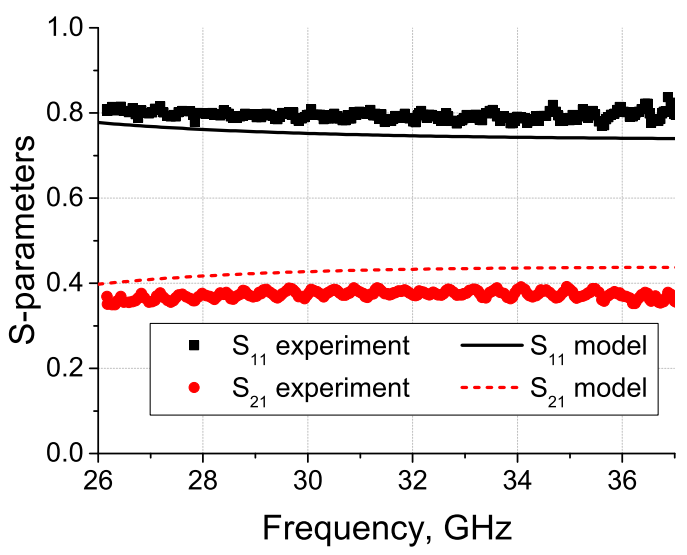

(a)

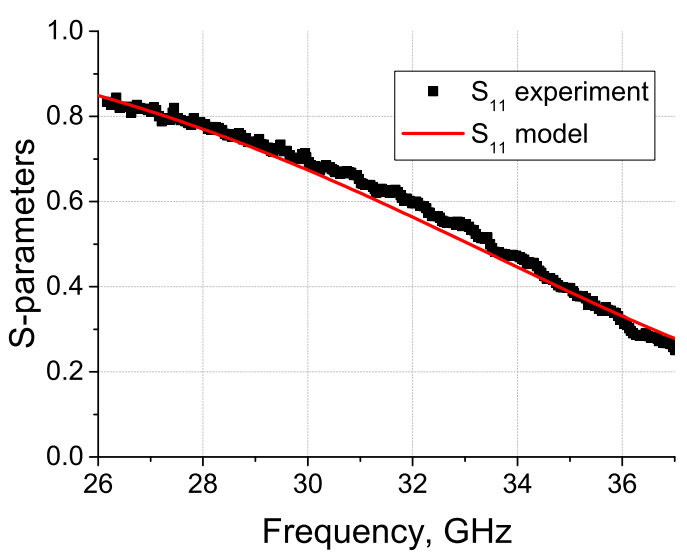

(b)

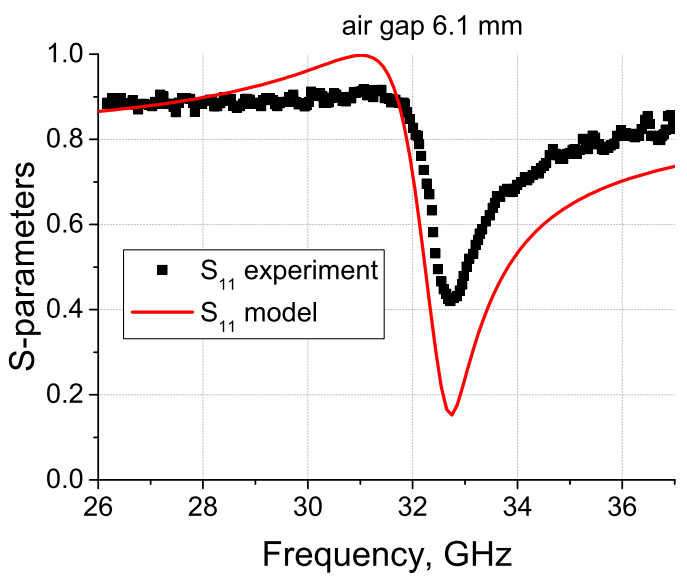

(c)

FIG. 7. Comparison of experimental (full circles and squares) and theoretical (lines) results in waveguide: (a) $S_{11^{-}}$and $S_{21}$-parameters of a freestanding 0.79-mm thick GCS-monolayer; (b) $S_{11}$-parameters of the same GCS monolayer located on a metal plate; (c) $S_{11}$-parameters of the GCS monolayer separated from the metal plate by an air gap of width $l=6.1 \mathrm{~mm}$.

\section{ABSORPTION PROPERTIES IN FREE SPACE}

The results presented above were obtained with waveguide-based measurements. These are indeed the most accurate and widely developed techniques for investigating electromagnetic properties in dielectric spectroscopy. However, the most important practical use of microwave absorbers is associated with the free space applications. Eqs. (4) and (5) can be also applied in free space but, in such a 
case, wave vectors $k_{z}=2 \pi / \lambda$ and $k_{2 z}=2 \pi \sqrt{\varepsilon} / \lambda$ should be used.

To investigate the absorption properties of GCS-based monolayers on a PEC plate at a normal incidence angle in free space, we varied parameters such as the diameter of the GCS and the width of the air gap between the monolayer and the PEC plate. Additionally, for better understanding of the absorption properties, we extended the modelling from the relatively narrow Ka-band to the frequency range from 1 up to $40 \mathrm{GHz}$. This allowed having an overall view of the expected microwave absorbing properties and estimating the optimal parameters for a further practical use of GCS metasurfaces.

It is worth noting that the diameter of GCSs is the most important geometrical parameter in the considered system. Additionally, air gap is the most flexible, "easy to change" parameter defining electromagnetic response. The variation of both parameters significantly affects the electromagnetic response of GCS monolayers and presents the general features of absorbing properties of GCS-monolayers in microwaves.

\section{A. Diameter dependence}

The dependence of the absorption coefficient of a GCSbased monolayer on frequency and diameter in free space is presented in Fig. 8.

It can be seen from Fig. 8 that the first absorption maximum (wherein absorption is $100 \%$ ) is shifted towards lower frequencies when the GCS diameter increases. Both the width and height of such a peak remain practically constant. For larger diameters, a second, wider maximum appears. This maximum may be used in practice for designing broadband absorbers.

It is interesting to compare those results with the absorbing properties of a lossy homogeneous plane parallel plate of thickness $\tau$ and permittivity $\varepsilon=1-i \sigma / \omega \varepsilon_{0}$ located on a PEC plate. In Fig. 8, the first maximum in the Ka-band can be achieved with spheres of diameter about $1 \mathrm{~mm}$. The modelling of a plane parallel plate with $\varepsilon=1-i \sigma / \omega \varepsilon_{0}$ shows that, in this case, the same first maximum may also be achieved in

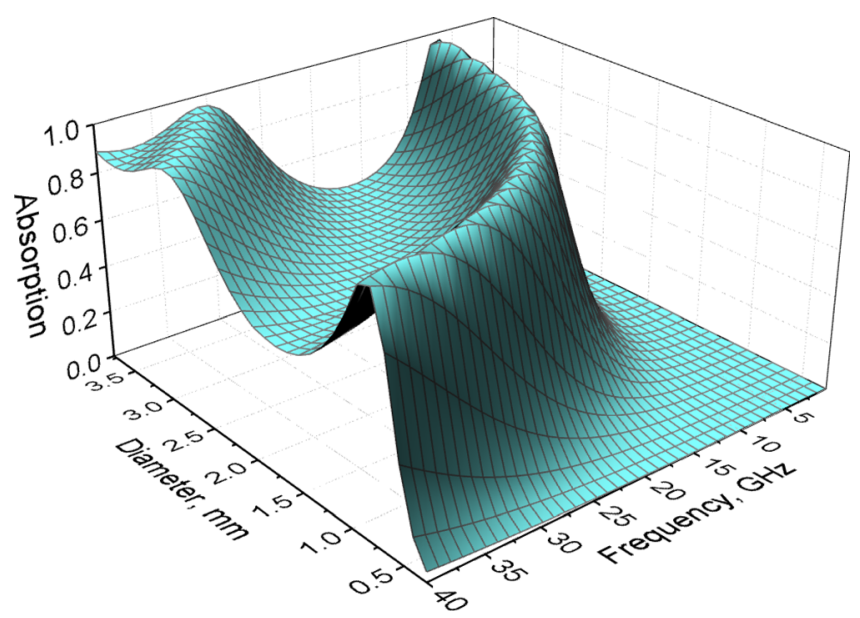

FIG. 8. Dependence of the absorption coefficient of the GCS-monolayer on frequency and diameter in free space. the Ka-band only for a minimal thickness of about 2.5-3 mm. The latter result shows that the GCS-based monolayer is much more compact than a plane parallel plate. Such an advantage of the GCS metasurface may be very important for aerospace applications for example.

Additionally, from the symmetry of the 3D-surface presented in Fig. 8 we can see that a change of diameter is equivalent to a change of frequency. This result is evident because, in the case of free space, Eq. (5) depends only on the simple product of thickness $\tau$ and frequency $\nu$. Therefore, the variations of both parameters in free space are equal. As in the case of hollow carbon spheres ${ }^{12}$ we see from Fig. 8 that for broadband absorption applications the spheres with a larger diameter are preferable.

\section{B. Air gap dependence}

The dependence of the absorption coefficient of a GCSbased monolayer on frequency and air gap width in free space is presented in Fig. 9.

Fig. 9 shows that the increase of the air gap makes the first absorption maximum decrease and be shifted to lower frequencies. The second maximum is clearly asymmetric, and sharper than the former one. This second maximum was exactly the one observed experimentally in the waveguide (Fig. 7(c)) at a frequency near $33 \mathrm{GHz}$. In Fig. 9, it is shifted to a lower frequency (near $26 \mathrm{GHz}$ ) due to the difference of wave vectors in free space and in a waveguide. Usually, if the interference absorption maximum is observed in the waveguide at a frequency $\nu_{w g}$, it is also expected in free space at a frequency $\nu_{f_{s}}$. In most cases, such a shift of frequency can be roughly estimated by the following equation: ${ }^{45}$

$$
\nu_{w g}^{2}-\nu_{f s}^{2}=\frac{c^{2}}{4 a^{2}},
$$

where $c$ is the speed of light in vacuum and $a$ is the width of the waveguide. At highest values of air gap, a third maximum also appears. The latter is even sharper than the second peak. The asymmetry and the variations between first, second, and third maxima are important differences between the GCS metasurface presented here and traditional lossy

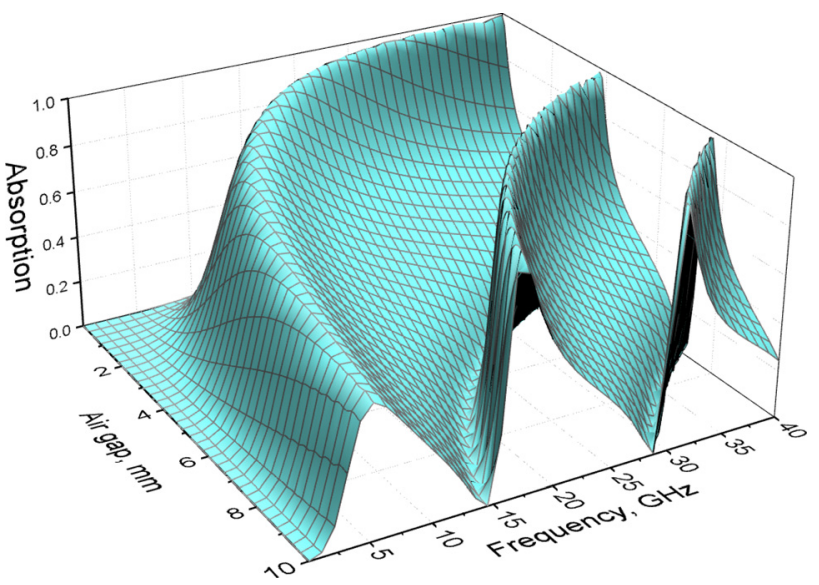

FIG. 9. Dependence of the absorption coefficient of the GCS-monolayer on frequency and air gap width in free space. 
homogeneous plane parallel plate of thickness $\tau$ and dielectric permittivity $\varepsilon=1-i \sigma / \omega \varepsilon_{0}$. In the case of a plane parallel plate, the first maximum indeed does not decrease with the air gap and the second and third maxima are symmetric and equivalent to the first one. We assume that this special feature of the GCS-based monolayer may find some practical applications in the future, for example for designing frequency-selective filters.

Also noteworthy is that the microwave absorption performances of the GCS monolayers considered here are generally comparable with those of other absorbers presented recently ${ }^{16-18}$ (the absorption mechanism is classical Ohmic losses in conductive medium). These recent reports generally deal with materials based on a polymer matrix and their operating temperature range is usually limited below $200-300^{\circ} \mathrm{C}$. In contrast, the glassy carbon considered here is thermally stable in air up to a temperature of $400{ }^{\circ} \mathrm{C}$, and even up to $3000{ }^{\circ} \mathrm{C}$ in vacuum. ${ }^{46}$

\section{CONCLUSIONS}

Porous glassy carbon spheres (GCSs), assumed to have a purely imaginary dielectric permittivity $\varepsilon=1-i \sigma / \omega \varepsilon_{0}$, were used for obtaining a microwave-absorbing metasurface. Spherical particles arranged in a periodic structure indeed allowed obtaining a metamaterial with graded real and imaginary parts of effective dielectric permittivity (see Fig. 6).

A detailed comparison of the absorption properties of the aforementioned GCS-based metasurface with those of a homogeneous lossy plane parallel plate of thickness $\tau=D$ and permittivity $\varepsilon=1-i \sigma / \omega \varepsilon_{0}$ located on a PEC plate led to the following differences, features, and advantages of the new heterostructure presented here:

- the experimental waveguide measurements showed that GCS monolayers absorb up to $92 \%$ of the initial microwave radiation power in the Ka-band;

- theoretical predictions showed that absorption peaks in free space may be effectively tuned by varying the width of the air gap and the diameter of the GCSs;

- the GCS monolayer is a compact absorber. The same absorbing parameters can indeed only be achieved with a conductive plane parallel plate presenting a significantly higher thickness;

- the carbon material presented in this manuscript may be applied as broadband or as a resonant absorber, depending on the width of the air gap;

- tuning the air gap allows obtaining asymmetric absorption peaks, which might be potentially used in microwave filter applications.

Finally, it is also worth mentioning that the material considered here is thermally stable, inert, easy to prepare, and cheap. These features of GCSs make them very attractive for designing new, effective microwave-absorbing coatings.

\section{ACKNOWLEDGMENTS}

The work was supported by Projects FP7-610875 (NAMICEMC, 2013-2017), H2020 RISE 734164 Graphene
3D, and FP7 IRSES project CANTOR (Grant No. FP7612285). Sijin Li thanks the China Scholarship Council for the financial support under Grant No. 201406510029. Cameron Gallagher and Emma Burgess acknowledge financial support from the Engineering and Physical Sciences Research Council (EPSRC) of the United Kingdom, via the EPSRC Centre for Doctoral Training in Metamaterials (Grant No. EP/L015331/1).

${ }^{1}$ J. B. Pendry, A. J. Holden, D. Robbins, and W. Stewart, "Magnetism from conductors and enhanced nonlinear phenomena," IEEE Trans. Microwave Theory Tech. 47, 2075-2084 (1999).

${ }^{2}$ D. R. Smith, W. J. Padilla, D. Vier, S. C. Nemat-Nasser, and S. Schultz, "Composite medium with simultaneously negative permeability and permittivity," Phys. Rev. Lett. 84, 4184 (2000).

${ }^{3}$ X. Liu, K. Fan, I. V. Shadrivov, and W. J. Padilla, "Experimental realization of a terahertz all-dielectric metasurface absorber," Opt. Express 25, 191-201 (2017).

${ }^{4}$ G. Tondi, V. Fierro, A. Pizzi, and A. Celzard, "Tannin-based carbon foams," Carbon 47, 1480-1492 (2009).

${ }^{5}$ F. Moglie, D. Micheli, S. Laurenzi, M. Marchetti, and V. M. Primiani, "Electromagnetic shielding performance of carbon foams," Carbon 50, 1972-1980 (2012).

${ }^{6}$ D. Bychanok, A. Plyushch, K. Piasotski, A. Paddubskaya, S. Voronovich, P. Kuzhir, S. Baturkin, A. Klochkov, E. Korovin, M. Letellier, S. Schaefer, A. Szczurek, V. Fierro, and A. Celzard, "Electromagnetic properties of polyurethane template-based carbon foams in Ka-band," Phys. Scr. 90, 094019 (2015).

${ }^{7}$ A. Szczurek, G. Amaral-Labat, V. Fierro, A. Pizzi, E. Masson, and A. Celzard, "The use of tannin to prepare carbon gels. Part I: Carbon aerogels," Carbon 49, 2773-2784 (2011).

${ }^{8}$ M. Inagaki and F. Kang, Materials Science and Engineering of Carbon: Fundamentals (Butterworth-Heinemann, 2014), p. 298.

${ }^{9}$ F. Walsh, L. Arenas, C. Ponce de Leon, G. Reade, I. Whyte, and B. Mellor, "The continued development of reticulated vitreous carbon as a versatile electrode material: Structure, properties and applications," Electrochim. Acta 215, 566-591 (2016).

${ }^{10}$ S. V. K. E. Zhuravlev and K. Dorozhkin, "Electromagnetic waves absorbing characteristics of composite material containing carbonyl iron particles," Mater. Sci. Appl. 5, 803-811 (2014).

${ }^{11}$ M. Ipatov, V. Zhukova, L. V. Panina, and A. Zhukov, "Ferromagnetic microwires composite metamaterials with tuneable microwave electromagnetic parameters," PIERS Proc. 5, 586-590 (2009).

${ }^{12}$ D. Bychanok, S. Li, A. Sanchez-Sanchez, G. Gorokhov, P. Kuzhir, F. Ogrin, A. Pasc, T. Ballweg, K. Mandel, A. Szczurek, V. Fierro, and A. Celzard, "Hollow carbon spheres in microwaves: Bio inspired absorbing coating," Appl. Phys. Lett. 108, 013701 (2016).

${ }^{13}$ M.-S. Cao, W.-L. Song, Z.-L. Hou, B. Wen, and J. Yuan, "The effects of temperature and frequency on the dielectric properties, electromagnetic interference shielding and microwave-absorption of short carbon fiber/silica composites," Carbon 48, 788-796 (2010).

${ }^{14}$ R. Buchner, J. Barthel, and J. Stauber, "The dielectric relaxation of water between 0C and 35C," Chem. Phys. Lett. 306, 57-63 (1999).

${ }^{15} \mathrm{~W}$. Withayachumnankul and D. Abbott, "Metamaterials in the terahertz regime," IEEE Photonics J. 1, 99-118 (2009).

${ }^{16}$ M. S. Sarto, A. G. D'Aloia, A. Tamburrano, and G. De Bellis, "Synthesis, modeling, and experimental characterization of graphite nanoplateletbased composites for emc applications," IEEE Trans. Electromagn. Compat. 54, 17-27 (2012).

${ }^{17}$ Y. Danlee, I. Huynen, and C. Bailly, "Thin smart multilayer microwave absorber based on hybrid structure of polymer and carbon nanotubes," Appl. Phys. Lett. 100, 213105 (2012).

${ }^{18}$ D. Bychanok, G. Gorokhov, D. Meisak, P. Kuzhir, S. A. Maksimenko, Y. Wang, Z. Han, X. Gao, and H. Yue, "Design of carbon nanotube-based broadband radar absorber for Ka-band frequency range," Prog. Electromagn. Res. 53, 9-16 (2017).

${ }^{19}$ C. L. Holloway, E. F. Kuester, J. A. Gordon, J. O'Hara, J. Booth, and D. R. Smith, "An overview of the theory and applications of metasurfaces: The two-dimensional equivalents of metamaterials," IEEE Antennas Propag. Mag. 54, 10-35 (2012).

${ }^{20}$ C. L. Holloway, E. F. Kuester, J. Baker-Jarvis, and P. Kabos, “A double negative (DNG) composite medium composed of magnetodielectric 
spherical particles embedded in a matrix," IEEE Trans. Antennas Propag. 51, 2596-2603 (2003).

${ }^{21}$ S. Kim, E. F. Kuester, C. L. Holloway, A. D. Scher, and J. Baker-Jarvis, "Boundary effects on the determination of metamaterial parameters from normal incidence reflection and transmission measurements," IEEE Trans. Antennas Propag. 59, 2226-2240 (2011).

${ }^{22} \mathrm{~A}$. Mejdoubi and C. Brosseau, "Dielectric response of perforated twodimensional lossy heterostructures: A finite-element approach,” J. Appl. Phys. 100, 094103 (2006)

${ }^{23}$ L. Lewin, "The electrical constants of a material loaded with spherical particles," J. Inst. Electr. Eng., Part III 94, 65-68 (1947).

${ }^{24}$ D. I. Kim, M. Takahashi, H. Anzai, and S. Y. Jun, "Electromagnetic wave absorber with wide-band frequency characteristics using exponentially tapered ferrite," IEEE Trans. Electromagn. Compat. 38, 173-177 (1996).

${ }^{25}$ E. F. Kuester and C. L. Holloway, "A low-frequency model for wedge or pyramid absorber arrays-I: Theory," IEEE Trans. Electromagn. Compat. 36, 300-306 (1994).

${ }^{26} \mathrm{H}$. J. Yoon and D. I. Kim, "Two-dimensional simulation of broad-band ferrite electromagnetic wave absorbers by using the fdtd method," J. Korean Phys. Soc. 45, 1025-1031 (2004).

${ }^{27}$ D. S. Bychanok, A. O. Plyushch, G. V. Gorokhov, U. S. Bychanok, P. P. Kuzhir, and S. A. Maksimenko, "Microwave radiation absorbers based on corrugated composites with carbon fibers," Tech. Phys. 61, 1880-1884 (2016).

${ }^{28}$ M.-J. Park, J. Choi, and S.-S. Kim, "Wide bandwidth pyramidal absorbers of granular ferrite and carbonyl iron powders," IEEE Trans. Magn. 36, 3272-3274 (2000).

${ }^{29}$ See http://niipfp.bsu.by/index.php/oborud/tora for "Microwave absorber "Tora"”.

${ }^{30}$ D. Stavenga, S. Foletti, G. Palasantzas, and K. Arikawa, "Light on the moth-eye corneal nipple array of butterflies," Proc. R. Soc. London, Ser. B 273, 661-667 (2006).

${ }^{31}$ H. A. Macleod, Thin-Film Optical Filters (CRC Press, 2001).

${ }^{32}$ N. Klochko, G. Khrypunov, Y. Myagchenko, E. Melnychuk, V. Kopach, K. Klepikova, V. Lyubov, and A. Kopach, "Electrodeposited zinc oxide arrays with the moth-eye effect," Semiconductors 48, 531-537 (2014).

${ }^{33}$ C.-H. Sun, P. Jiang, and B. Jiang, "Broadband moth-eye antireflection coatings on silicon,” Appl. Phys. Lett. 92, 061112 (2008).
${ }^{34}$ S. F. Parker, S. Imberti, S. K. Callear, and P. W. Albers, "Structural and spectroscopic studies of a commercial glassy carbon," Chem. Phys. 427, 44-48 (2013).

${ }^{35}$ M. Letellier, A. Szczurek, M.-C. Basso, A. Pizzi, V. Fierro, O. Ferry, and A. Celzard, "Preparation and structural characterisation of model cellular vitreous carbon foams," Carbon 112, 208-218 (2017).

${ }^{36}$ L. Soukup, I. Gregora, L. Jastrabik, and A. Konakova, "Raman spectra and electrical conductivity of glassy carbon,” Mater. Sci. Eng., B 11, 355-357 (1992).

${ }^{37}$ K. Ray and R. L. McCreery, "Spatially resolved Raman spectroscopy of carbon electrode surfaces: Observations of structural and chemical heterogeneity," Anal. Chem. 69, 4680-4687 (1997).

${ }^{38}$ S. Bukalov, L. Leites, A. Sorokin, and A. Kotosonov, "Structural changes in industrial glassy carbon as a function of heat treatment temperature according to Raman spectroscopy and x-ray diffraction data," Nanosyst. Phys. Chem. Math. 5, 186-191 (2014).

${ }^{39}$ N. Solopova, N. Dubrovinskaia, and L. Dubrovinsky, "Raman spectroscopy of glassy carbon up to $60 \mathrm{GPa}$,” Appl. Phys. Lett. 102, 121909 (2013).

${ }^{40}$ M. Nakamizo, R. Kammereck, and P. L. Walker, "Laser Raman studies on carbons," Carbon 12, 259-267 (1974).

${ }^{41}$ P. Mallet-Ladeira, P. Puech, C. Toulouse, M. Cazayous, N. Ratel-Ramond, P. Weisbecker, G. L. Vignoles, and M. Monthioux, "A Raman study to obtain crystallite size of carbon materials: A better alternative to the Tuinstra-Koenig law," Carbon 80, 629-639 (2014).

${ }^{42}$ F. Cuevas, J. Montes, J. Cintas, and P. Urban, "Electrical conductivity and porosity relationship in metal foams," J. Porous Mater. 16, 675 (2009).

${ }^{43}$ D. Micheli, R. B. Morles, M. Marchetti, F. Moglie, and V. M. Primiani, "Broadband electromagnetic characterization of carbon foam to metal contact," Carbon 68, 149-158 (2014).

${ }^{44}$ M. Born and E. Wolf, Principles of Optics, 4th ed. (Pergamon Press, 1970).

${ }^{45}$ D. Bychanok, A. Plyushch, G. Gorokhov, V. Skadorov, P. Kuzhir, S. Maksimenko, J. Macutkevic, A. Ortona, L. Ferrari, and E. Rezaei, "Electromagnetic properties of periodic carbon architectures at high frequencies," in 2015 International Conference on Electromagnetics in Advanced Applications (ICEAA) (IEEE, 2015), pp. 43-46.

${ }^{46}$ F. C. Cowlard and J. C. Lewis, "Vitreous carbon - A new form of carbon," J. Mater. Sci. 2, 507-512 (1967). 